\title{
Technè
}

La science au service de l'histoire de l'art et de la préservation des biens culturels

41 | 2015

Arts textiles antiques et modernes. Approche scientifique et restauration

\section{Les fondations mésopotamiennes, conservatoire pour l'étude des textiles}

Mesopotamian foundations, a conservatory for the study of textiles

\section{Ariane Thomas et Christophe Moulherat}

\section{(2) OpenEdition \\ 12 Journals}

Édition électronique

URL : http://journals.openedition.org/techne/4087

DOI : 10.4000/techne.4087

ISSN : 2534-5168

Éditeur

C2RMF

\section{Édition imprimée}

Date de publication : 25 novembre 2015

Pagination : 8-18

ISBN : 978-2-7118-6248-1

ISSN : 1254-7867

Référence électronique

Ariane Thomas et Christophe Moulherat, «Les fondations mésopotamiennes, conservatoire pour l'étude des textiles », Technè [En ligne], 41 | 2015, mis en ligne le 08 août 2020, consulté le 11 mars 2021. URL : http://journals.openedition.org/techne/4087 ; DOI : https://doi.org/10.4000/techne.4087

\section{(c)}

La revue Technè. La science au service de l'histoire de l'art et de la préservation des biens culturels est mise à disposition selon les termes de la Licence Creative Commons Attribution - Pas d'Utilisation Commerciale - Pas de Modification 4.0 International. 


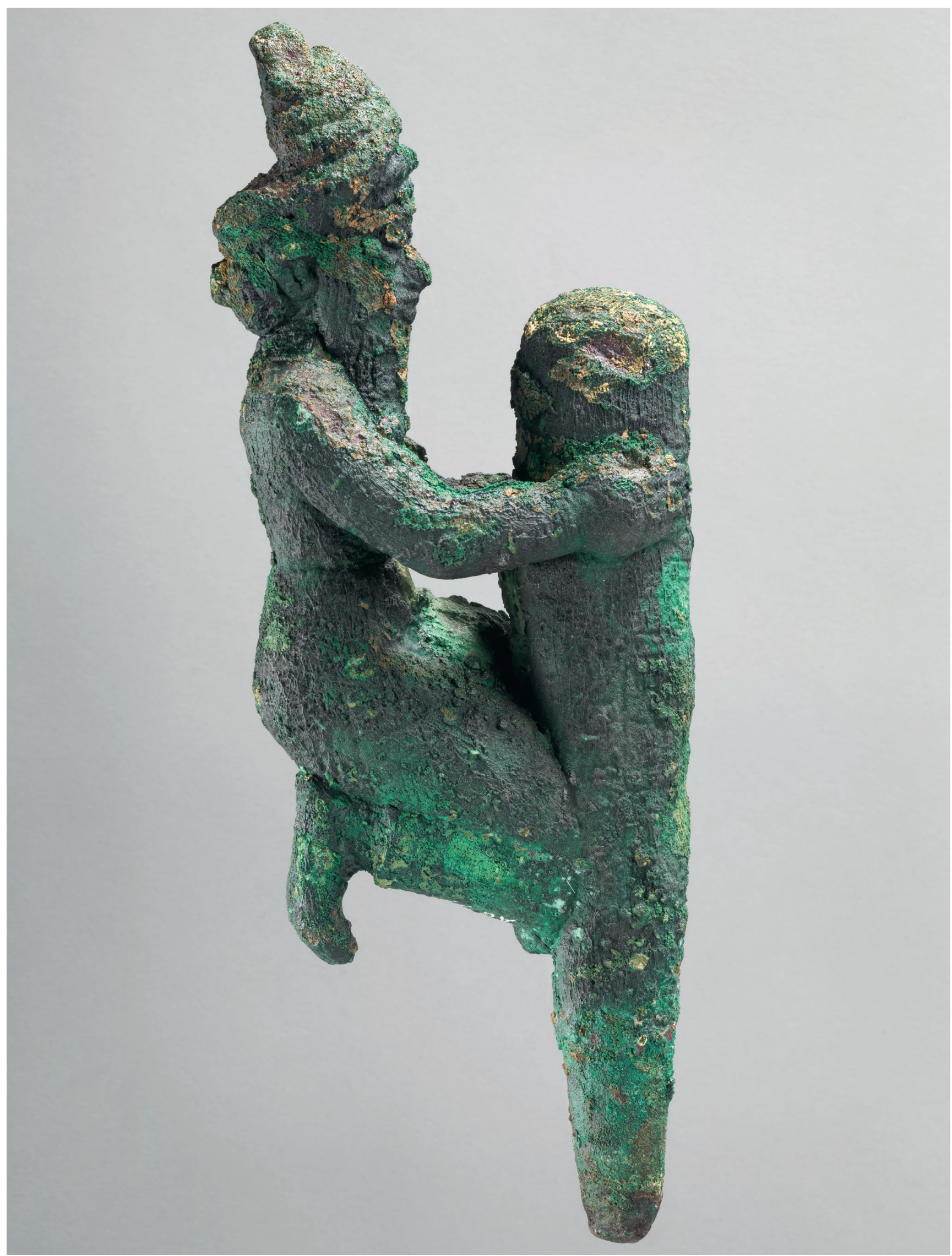

Fig. 1 a. Vue générale du clou de fondation. Musée du Louvre, département des Antiquités orientales, inv. AO 77. (c) Musée du Louvre/Philippe Fuzeau. 
Ariane Thomas

Christophe Moulherat
Les fondations mésopotamiennes, conservatoire pour l'étude des textiles

Mesopotamian foundations, a conservatory

for the study of textiles

Résumé. Le département des Antiquités orientales du musée du Louvre conserve des objets sur lesquels subsistent des textiles sous une forme minéralisée qui font actuellement l'objet d'une étude globale inédite. Nous présentons ici l'étude des textiles conservés sur les clous de fondation de la II ${ }^{e}$ dynastie de Lagash ( $f$ in du III ${ }^{e}$ millénaire avant J.-C. ; Mésopotamie méridionale).

Mots-clés. Clou de fondation, néo-sumérien, Lagash, Mésopotamie, textile, minéralisation, lin.
Abstract. Textiles in a mineralized form have survived on some of the objects found in the Louvre's Department of Near-Eastern Antiquities. These pieces are now the subject of an unprecedented worldwide study. In this article, we present the study of the textiles remaining on foundation nails dating from the Second Dynasty of Lagash (late third millennium BC, southern Mesopotamia).

Keywords. Foundation nail, Neo-Sumerian, Lagash, Mesopotamia, textile, mineralization, linen.

\section{Introduction}

Il était d'usage dans l'ancienne Mésopotamie que les temples et autres bâtiments d'importance soient consacrés par certains rituels incluant notamment divers dépôts (tablettes, figurines, ensembles d'objets) dans les fondations. Du III ${ }^{\mathrm{e}}$ au I ${ }^{\mathrm{er}}$ millénaire avant J.-C., ces dépôts étaient rituellement enfouis au moment de la construction puis ré-enfouis lors des rénovations successives. L'un des dépôts les plus courants prend la forme d'un clou$^{1}$. Apparus au milieu du III' millénaire avant notre ère, ces clous sont en terre cuite ou en métal, soit sous une forme simple, soit figurés en homme ou en animal. Le propos de cet article se concentre sur un ensemble précis de clous de fondation datant de la $\mathrm{II}^{\mathrm{e}}$ dynastie de Lagash, royaume de Mésopotamie méridionale (dans le sud de l'actuel Irak), à l'époque dite néo-sumérienne, dont le plus fameux souverain est Gudea, vers 2100 avant J.-C. Plusieurs de ces clous conservent en effet d'exceptionnels vestiges textiles (fig. 1 a et b) qui ont fait l'objet d'une étude systématique, à la fois technique et historique, dont nous présentons ici les résultats.

\section{De la fouille au musée}

Chacun des clous de fondation de la $\mathrm{II}^{\mathrm{e}}$ dynastie de Lagash était systématiquement associé à une tablette inscrite en pierre. Également inscrits, les clous, toujours en cuivre plein ${ }^{2}$, se déclinaient en trois variantes ${ }^{3}$ :
- figurant un dieu barbu, coiffé d'une tiare à cornes (caractérisant les divinités depuis l'époque d'Akkad) et vêtu d'un pagne court et d'un habit long ouvert sur le côté gauche ; il se tient accroupi, un genou à moitié relevé, et maintient de ses deux mains un clou placé entre ses jambes pour le ficher dans le sol ;

- surmonté d'un homme imberbe ${ }^{4}$ debout portant une corbeille à briques ;

- surmonté d'un taureau agenouillé au-dessus d'un clou.

Ils ont été mis au jour sur le site de Tello (cité de l'antique royaume de Lagash) durant les fouilles d'Ernest de Sarzec entre 1877 et 1900, de Gaston Cros (1903-1909), d'Henri de Genouillac (1929-1931) et d'André Parrot (1931-1933). Le musée du Louvre en conserve 17 sur un total actuellement connu de 40 clous de fondation de cette dynastie, dont $36 \mathrm{du}$ type du dieu agenouillé ${ }^{5}$ (voir tableaux I et II).

Parmi ces clous, au moins $14^{6}$ portaient des vestiges textiles visibles à l'œil nu au moment de leurs découvertes, comme en attestent tant les écrits des fouilleurs ${ }^{7}$ que quelques photographies et fiches anciennes du musée. Douze ${ }^{8}$ clous en conservent aujourd'hui tandis que les autres les ont perdus depuis (tableau I) : au moment de la fouille ou à leur arrivée au musée, mais aussi plus tard lors de malencontreuses restaurations ${ }^{9}$. Il est vrai qu'il est difficile d'intervenir sur ces objets fragiles sans porter atteinte à leur surface alors même que leur corrosion parfois très active rend nécessaire un traitement. C'est pourtant leur dégradation qui a permis de conserver ces

Dr. Ariane Thomas, conservateur du patrimoine en charge des collections mésopotamiennes, département des Antiquités orientales, musée du Louvre (ariane.thomas@louvre.fr). Christophe Moulherat, chargé d'analyses des collections/référent textile, musée du quai Branly (christophe. moulherat@quaibranly.fr) 
textiles par la migration des sels métalliques du clou vers la surface, où ils ont piégé les matières organiques.

Étant donné l'état des textiles et du métal, il semble que le processus de minéralisation ait commencé peu après l'enfouissement des figurines ${ }^{10}$. Les textiles seraient donc bien contemporains de la II $^{\mathrm{e}}$ dynastie de Lagash quand bien même les textes évoquent l'usage d'exhumer les dépôts antérieurs lors de la restauration des temples jusqu'au I ${ }^{\text {er }}$ millénaire avant J.-C. ${ }^{11}$ En outre, le royaume de Lagash perdit son importance à la fin du $\mathrm{III}^{\mathrm{e}}$ millénaire après quoi les restaurations et reconstructions ne furent plus de mise.

Sachant que nous n'avons pu examiner que ceux du musée du Louvre, 12 clous sur les 17 de la collection ont assurément porté des textiles ${ }^{12}$, ainsi qu'au moins deux autres au Musée archéologique d'Istanbul (tableau I). Dès lors, il est permis de penser que l'ensemble des clous de fondation portait des tissus (tableau II). En outre, plusieurs clous de fondation à peu près contemporains mais provenant de Nippur et rattachés à la III ${ }^{\mathrm{e}}$ dynastie d'Ur, portent également des textiles encore bien visibles ${ }^{13}$. Il pourrait donc s'agir d'une pratique systématique, du moins à l'époque néo-sumérienne ; les textes cunéiformes ne le mentionnent pas, peut-être parce que l'habillement ou l'enveloppement des figurines de fondation était justement évident ${ }^{14}$. On peut s'interroger sur les raisons ou le hasard grâce auxquels certains ont été épargnés, mais les textiles ont longtemps été peu considérés, sans doute en partie parce que l'on ne pouvait aller au-delà d'hypothèses sans les outils qui permettent à présent d'étudier ces vestiges.

\section{Un travail inédit en cours}

Entamée en $2012^{15}$, l'étude technique et historique la plus complète possible a été menée sur les clous de la $\mathrm{II}^{\mathrm{e}}$ dynastie de Lagash au musée du Louvre. Nous en présentons ici la méthode et les résultats ainsi que des questions en suspens. Dans le prolongement de ce travail sur les clous, nous avons établi le corpus complet des textiles conservés au département des Antiquités orientales ${ }^{16}$. Ce travail inédit contredit l'idée selon laquelle les textiles et autres restes organiques seraient presque totalement perdus pour la Mésopotamie et plus largement l'Orient ancien. S'il est exact que les conditions géo-climatiques et la nature des sols sont défavorables à leur conservation, notre travail démontre l'existence de corpus textiles cohérents dont l'étude est possible ${ }^{17}$. Replacés dans ce cadre élargi, les textiles minéralisés à la surface des clous de fondation peuvent nous en apprendre davantage sur les textiles de cette époque et les rites de fondation à Lagash et en Mésopotamie.

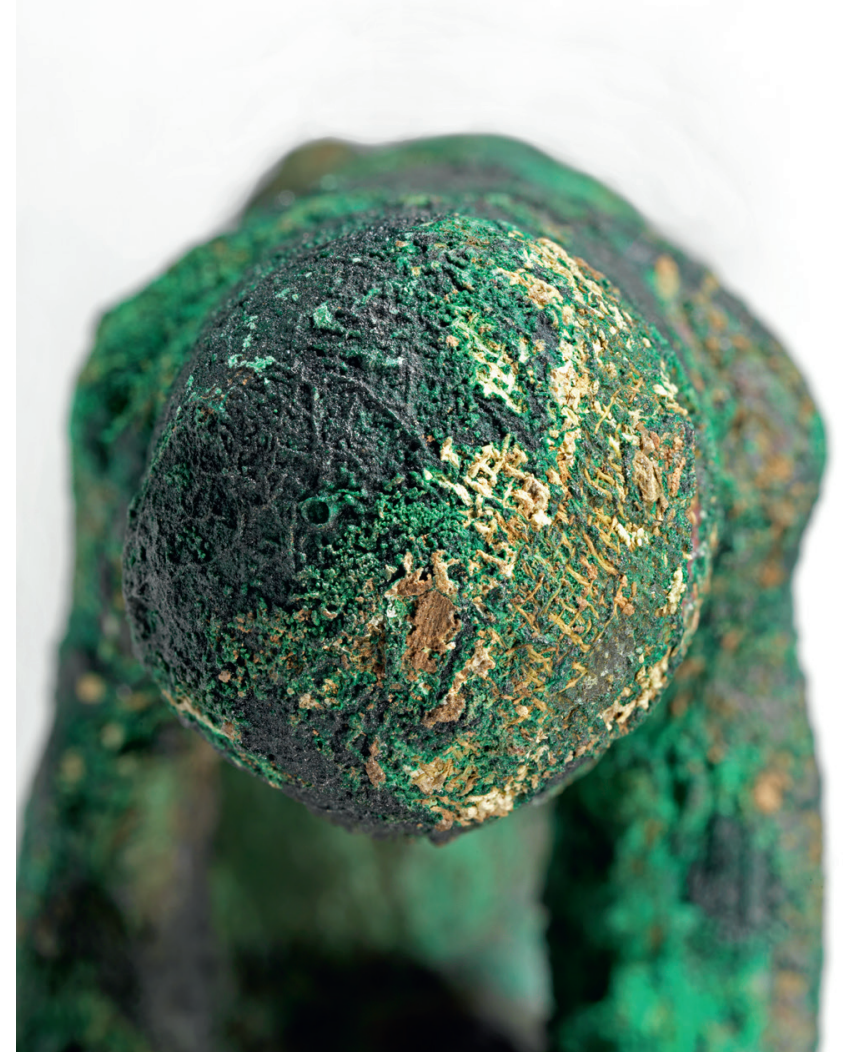

Fig. 1 b. Vue détaillée du sommet du clou. Musée du Louvre, département des Antiquités orientales, inv. AO 77.

๑) Musée du Louvre/Philippe Fuzeau.

\section{Des textiles : méthode et résultats}

Chaque clou a d'abord été observé à l'aide d'un microscope portatif et d'une loupe binoculaire. Les clous inventoriés AO 76 et AO 77 (fig. 1 a et b) ont également pu être observés sous microscope numérique 3D Hirox au Centre de recherches et de restauration des musées de France $(\mathrm{C} 2 \mathrm{RMF})^{18}$. Enfin, les 3 clous en forme de dieu agenouillé portant des vestiges textiles suffisamment conservés ont fait l'objet d'un prélèvement ${ }^{19}$, examiné sous stéréomicroscope et microscope électronique à balayage (MEB) suivant la méthode décrite ci-dessous.

\section{Méthode}

La diversité des formes d'altération des textiles archéologiques nuit considérablement à leur étude qui se limite le plus souvent à l'examen de leur texture. En outre, leur nature minérale ne convient pas aux méthodes traditionnelles d'identification (microscopie optique à transmission). Afin de combler cette lacune, une nouvelle méthode d'identification a été mise au point ${ }^{20}$. S'appuyant sur une connaissance approfondie de nombreux échantillons très divers de fibres souples et minéralisées, cette méthode combine la microscopie électronique à balayage et la microscopie optique. La préparation des 
échantillons est spécifique aux fibres minéralisées. Pour les vues longitudinales, on procède au prélèvement d'un échantillon dont la taille n'excède pas $5 \mathrm{~mm}^{2}$ avant de l'observer au MEB. Pour les vues transversales, l'échantillon est inclus dans un bloc de résine polyester transparente (Sody 33). Après une polymérisation dont la durée n'excède pas 24 heures, il est finement poli. La coupe obtenue est observée au microscope optique en réflexion. Il convient de choisir judicieusement le plan de coupe pour déterminer la nature des fibres.

L'originalité de cette approche tient à la préparation des coupes transversales ${ }^{21}$, combinée à la double observation aux microscopes électronique et optique. Les observations obtenues, d'excellente qualité, permettent de déterminer la nature des fibres, chacune d'elles possédant ses propres caractéristiques (forme et importance des écailles, diamètre des fibres, présence ou non d'un lumen, forme du lumen...). Il est également possible d'apprécier la qualité des techniques de filage et de tissage.

\section{Résultats}

Le $\mathrm{MEB}^{22}$ a permis d'observer la présence de genoux de flexion et de surface facettée, spécifique aux fibres libériennes (lin, chanvre, ramie, jute). Des observations en coupe transversale ont permis de préciser qu'il s'agit de lin puisque la section de ces fibres est polygonale avec des angles plutôt arrondis. En outre, la plupart d'entre elles sont isolées et non regroupées en faisceau, comme c'est le cas de la plupart des fibres libériennes mais pas des fibres de lin qui ont tendance à se dissocier les unes des autres, la lamelle mitoyenne de chacune d'elles étant peu lignifiée.

Les vestiges analysés (tableau I) sont donc tous en lin (fig. 3). Or à l'époque néo-sumérienne, la Mésopotamie fut grande productrice de laine ${ }^{23}$, au regard de laquelle la production d'étoffes de lin représentait moins de $10 \%$ d'après les archives cunéiformes ${ }^{24}$. Le lin était donc plus rare que les tissus de laine, voire importé du Levant ou d'Égypte ${ }^{25}$. Il est d'ailleurs attesté dans la garde-robe des rois comme des dieux ${ }^{26}$ mais, sachant qu'il existait aussi des laines de grand prix, la valeur du lin - d'origine végétale et non animale - dépassait peut-être sa préciosité, d'autant qu'il a été utilisé pour les objets hautement symboliques que sont les clous de fondation. À ce titre, il est notable que sur le seul exemple suffisamment conservé (fig. 2), le lin apparaisse de sa couleur naturelle ${ }^{27}$, à savoir une teinte claire dont la blancheur constituait, en Mésopotamie, une des composantes de sa valeur ${ }^{28}$, peut-être synonyme de pureté. En outre, ce lin était de grande qualité d'après le lumen qui est petit et très fin (diamètre entre 8 et $20 \mu \mathrm{m}$, la moyenne étant le plus souvent autour de 18 ; la fibre élémentaire a un diamètre de $8 \mu \mathrm{m}$ et reste difficile à observer). On remarque également que toutes les étapes de traitement de la fibre étaient parfaitement maîtrisées (rouissage, teillage, peignage), compte tenu de la finesse des fils et de la présence de petits faisceaux et de fibres isolées.

À l'exception d'un exemple combinant fils retors de torsion $\mathrm{S}$ de deux bouts $\mathrm{Z}$ et fils simples de torsion $\mathrm{Z}$, les vestiges examinés sont tous tissés suivant une armure toile constituée
Fig. 2. Vue détaillée au microscope du textile situé au bas de la barbe du dieu. Musée du Louvre, département des Antiquités orientales, inv. AO 77. (C) C2RMF/ Dominique Robcis.

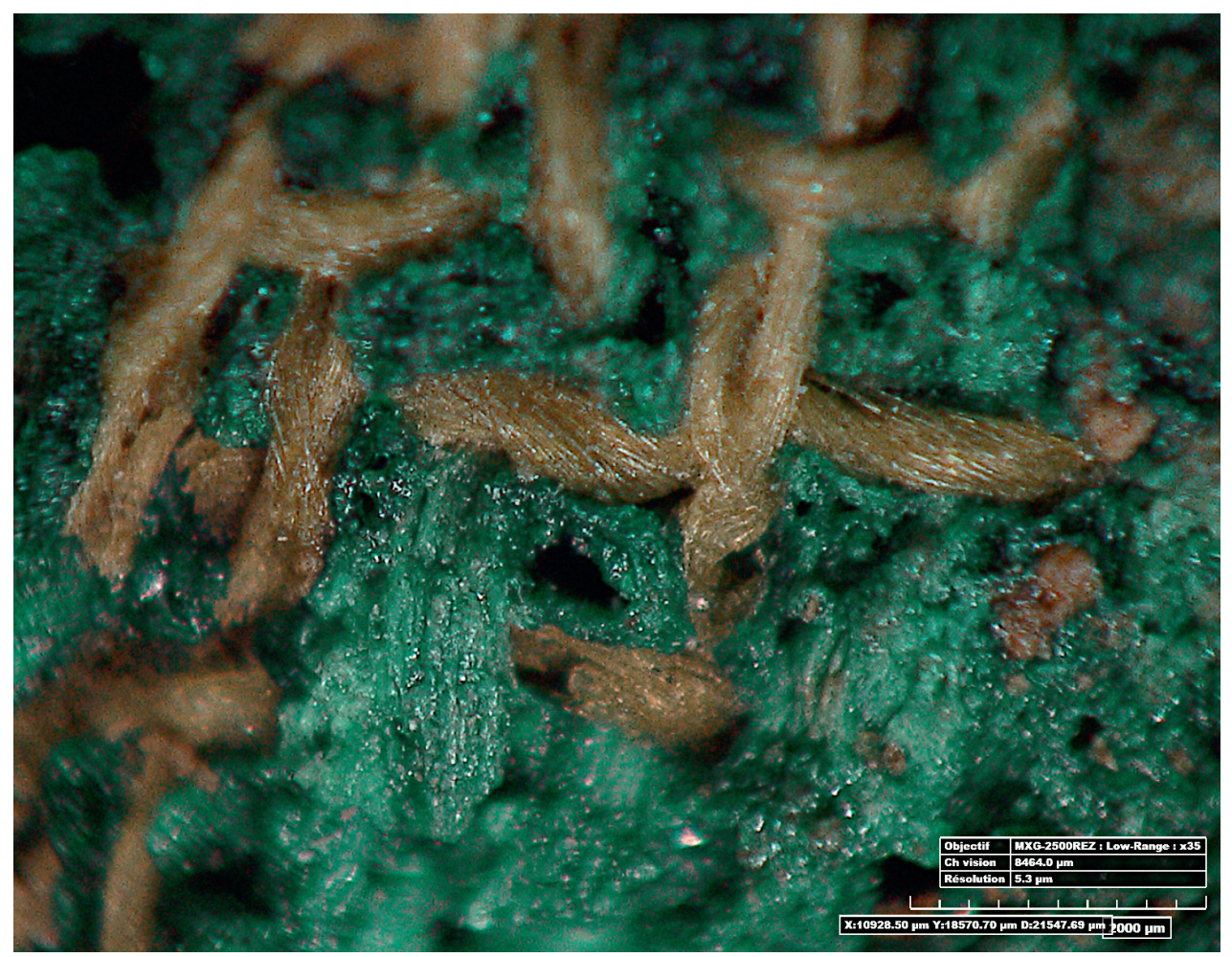




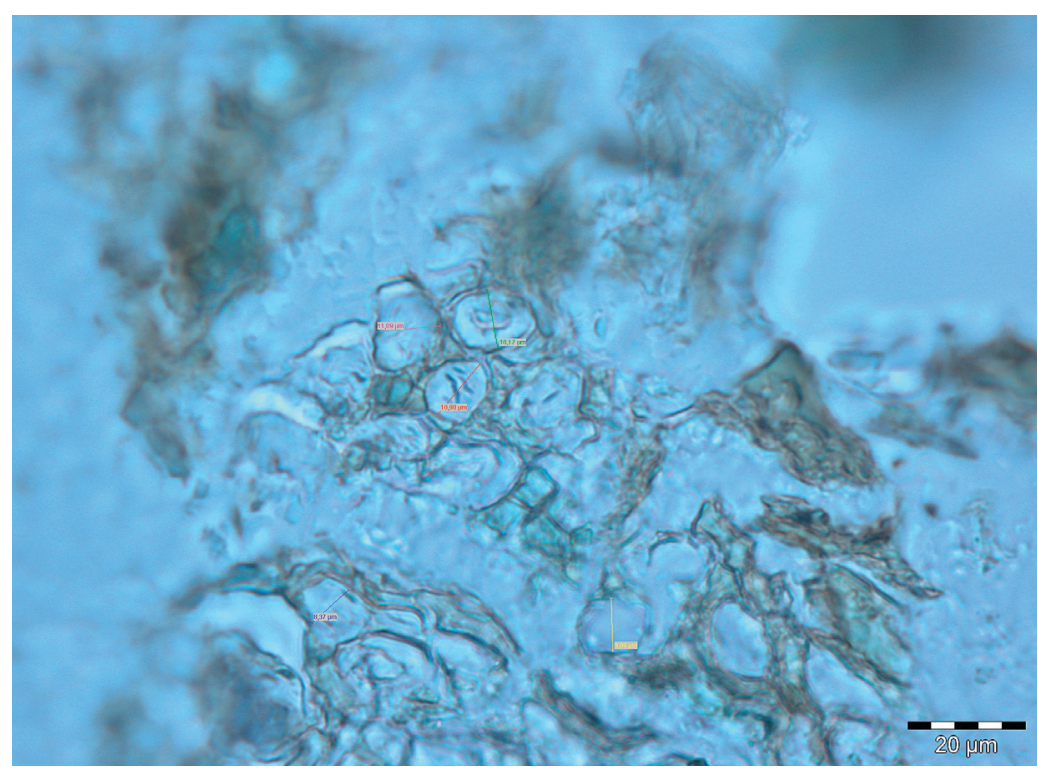

Fig. 3. Vue du prélèvement au microscope électronique à balayage. Musée du Louvre, département des Antiquités orientales, inv. AO 77. (c) Christophe Moulherat.

de fils retors de torsion $\mathrm{S}$ de deux bouts $\mathrm{Z}$, comptant entre 15 et 20 fils par cm en moyenne (tableau I). L'armure est dite équilibrée dans la mesure où elle ne présente pas de différence - sinon très légère - entre le nombre de fils dans les deux directions, sans que l'on puisse distinguer la trame de la chaîne, autant visibles l'une que l'autre. Cette armure est relativement dense et le tissu adhère bien, sans déformation, aux formes de l'objet, ce qui conforte l'idée que l'étoffe était de qualité. En fait, tous ces tissus partagent une même base de fils simples de torsion $\mathrm{Z}$, remarquablement fins ${ }^{29}$ (diamètre : 0,15-0,20 mm). Certains vestiges, dont un seul suffisamment bien conservé pour être fiable (AO 77 ; AO 312 (?) ; MNB 1362 (?)), comportent des fils simples tels quels. Mais, dans la plupart des cas, ces fils de base furent combinés par deux en torsion $\mathrm{S}$, ce qui double le diamètre moyen des fils entre 0,30 et 0,40 mm (AO 76 ; MNB 1365 ; MNB 1374 ; MNB 1377 ; MNB 1384). Les raisons du choix de telle ou telle combinaison entre fils simples $\mathrm{Z}$ et retors $\mathrm{S}$ de deux bouts $\mathrm{Z}$ nous échappent tout à fait.

\section{Des filaments}

Sous les vestiges textiles, d'autres traces minéralisées plus énigmatiques ${ }^{30}$ - que nous appelons filaments - sont visibles à la surface des clous de fondation ${ }^{31}$ (fig. 4$)^{32}$. Elles se présentent de manière relativement ordonnée, sinuant l'une contre l'autre en suivant les grandes lignes de l'objet. Par endroits, notamment sous les bras ou au niveau des pieds, elles semblent plus épaisses. Il ne saurait s'agir ni de feutre ${ }^{33}$, ni d'un faciès de corrosion ${ }^{34}$. Il semble également peu probable que ce soient les seules traces d'un enduit ou d'une huile parfumée appliquée à l'objet avant le textile et l'enfouissement de l'ensemble, comme les textes de la II ${ }^{\mathrm{e}}$ dynastie de Lagash sur les rites de fondation en témoignent ${ }^{35}$. Ces traces pourraient correspondre à une réaction de l'enduit et du textile, voire à une solution appliquée au tissu lui-même ${ }^{36}$. À ce titre, la souplesse du tissu adhérant aux formes de l'objet pourrait être liée à la présence d'un produit qui l'aurait imprégné.

\section{Vêtement ou enveloppe?}

Les étoffes conservées étaient-elles destinées à envelopper ou à habiller ces clous ? La question se pose tout particulièrement pour les clous figurant des dieux agenouillés et des porteurs de corbeille. Les textes indiquent en effet que l'on habillait les statues divines et royales ${ }^{37}$. Or le dieu agenouillé, d'après le nombre de cornes de sa tiare, était une divinité majeure qui méritait tous les égards. Il pourrait s'agir de Ningirsu, le dieu personnel de Gudéa ${ }^{38}$, ou d'Enki, le grand dieu démiurge des eaux et de la sagesse ${ }^{39}$. Quant aux porteurs de corbeille, ils représentent vraisemblablement le roi, comme semblent le confirmer plusieurs textes cunéiformes ${ }^{40}$. Or ces textes laissent percevoir que, bien qu'enfouis sous terre et ne recevant donc pas de ration régulière de nourriture et vêtement, ces clous étaient néanmoins des incarnations magiques. À ce titre, la nudité étant synonyme de faiblesse en Mésopotamie, ces incarnations devaient peut-être être habillées, d'autant plus que les porteurs de corbeille ont le torse nu tandis que les dieux ont une épaule découverte et que l'on sait que certaines bêtes, notamment les taureaux, pouvaient être parées ${ }^{41}$.

La tête et le clou portent des traces de textile qui n'auraient pas lieu d'être s'il s'agissait d'habiller la figure. Cela soutient l'idée d'une enveloppe textile ${ }^{42}$ protégeant l'objet magique précieux, dans sa logette parfois elle-même revêtue d'une natte ${ }^{43}$. L'étude des autres textiles du département démontre d'ailleurs que de nombreux objets en métal (vases, 
outils, armes) étaient également enveloppés, de même que des tablettes en argile d'après certaines empreintes ${ }^{44}$. Par ailleurs, tant les dépôts retrouvés que les textes attestent de dépôts de textile, de bijoux ou de nattes, associés mais indépendants des figurines de fondation ${ }^{45}$.

L'onction combinée à l'enveloppement ou à l'habillement des figurines, ces dernières étant associées à des dépôts qui pourraient évoquer des offrandes funéraires, n'est pas sans rappeler l'ensevelissement d'un défunt.

\section{Conclusion}

L'étude de ces textiles a permis de mettre en avant la qualité du travail des fils de lin et de distinguer deux ensembles parmi ces tissages simples relativement homogènes : le premier, très majoritaire, se caractérise par l'usage exclusif de fils retors de torsion $\mathrm{S}$ dans les deux sens; le second montre l'usage de fils retors de torsion $\mathrm{S}$ dans un sens et de fils simples de torsion $\mathrm{Z}$ dans l'autre, dont le diamètre fait approximativement moitié moins que le diamètre des fils du premier groupe. En croisant ces données techniques inédites avec les données archéologiques et épigraphiques, les textiles conservés sur les clous de la $\mathrm{II}^{\mathrm{e}}$ dynastie de Lagash révèlent un pan sans doute systématique et, à ce titre, essentiel des rites de fondation, qui se trouvaient au cour de la vie publique et religieuse mésopotamienne. Ils témoignent de l'importance accordée aux textiles dans les rites et dans la symbolique mésopotamienne tout en étant les rares vestiges directs des industries textiles dont on connaît la place majeure dans l'économie mésopotamienne, notamment à la fin du III ${ }^{\mathrm{e}}$ millénaire avant J.-C.
Habillant ou enveloppant les figurines « magiques » de fondation, ces textiles pouvaient être à la fois protecteurs, marques de statut et garants de pureté avec d'autres éléments de purification et de protection tels que la ou les substances ayant servi à oindre ces figurines et dont l'association pourrait être à l'origine des filaments présents sous le textile. Grâce à des collaborations pluridisciplinaires, notre projet devrait désormais permettre de mieux comprendre les processus de minéralisation de ces textiles, auxquels il faudrait prêter davantage attention tant en fouilles que dans les musées susceptibles d'en conserver.

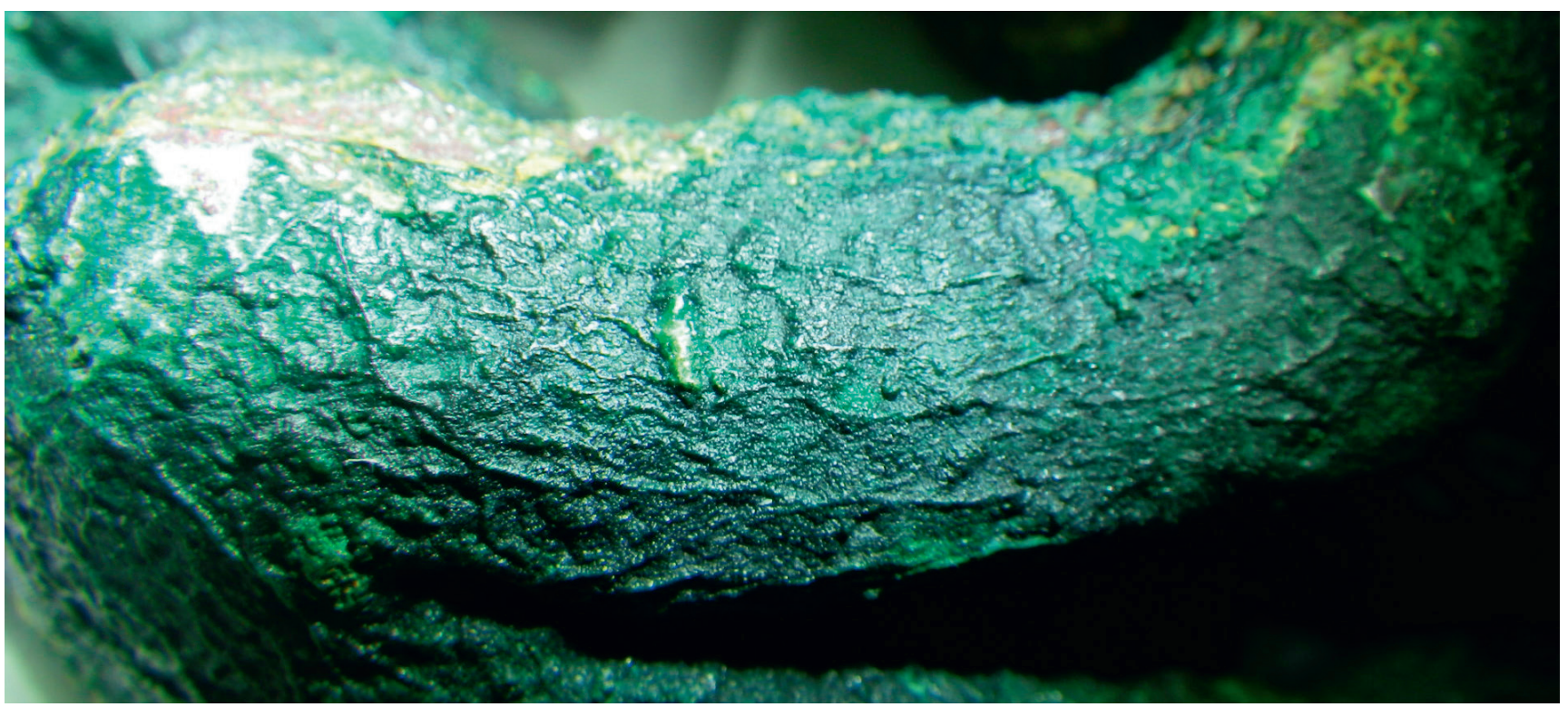

Fig. 4. Vue détaillée des filaments au niveau du bras droit. Musée du Louvre, département des Antiquités orientales, inv. AO 77. (c) Musée du Louvre/Ariane Thomas. 
Tableau I. Clous de fondation de la $\mathrm{II}^{\mathrm{e}}$ dynastie de Lagash avec vestiges textiles avérés (tous proviennent de Tello) (en italique: vestiges textiles attestés à leur découverte mais aujourd'hui disparus)

\begin{tabular}{|c|c|c|c|c|c|c|c|c|c|}
\hline & $\mathbf{N}^{\circ}$ d'inventaire & $\begin{array}{l}\text { Type de } \\
\text { clou de } \\
\text { fondation }\end{array}$ & $\begin{array}{l}\text { Dimensions } \\
\text { en cm }\end{array}$ & $\begin{array}{l}\text { Remarques/observations } \\
\text { dans le cadre de cette } \\
\text { étude }\end{array}$ & Textile/ état & Armure & Torsion de fils & Diamètre & Réduction \\
\hline \multicolumn{10}{|c|}{ Musée du Louvre, Paris } \\
\hline 1 & $\begin{array}{l}\text { AO } 75 \\
=83 ; \text { CAC }^{46} \\
156 ; \text { Tello } 431\end{array}$ & $\begin{array}{l}\text { porteur } \\
\text { de corbeille }\end{array}$ & $\begin{array}{l}\text { H. } 24,2 ; \\
\text { l. } 6,85 \\
\text { e. } 3,45\end{array}$ & $\begin{array}{l}\text { Textile aujourd'hui } \\
\text { disparu (sauf vestiges } \\
\text { infimes (?) à gauche, à } \\
\text { droite et à l'arrière de la } \\
\text { tête dans les creux); tissu } \\
\text { mentionné dans fiches et } \\
\text { photographies anciennes }\end{array}$ & Disparu & & & & \\
\hline 2 & $\begin{array}{l}\text { AO } 76 \\
=84 ; \\
\text { CAC } 154\end{array}$ & $\begin{array}{l}\text { dieu } \\
\text { agenouillé }\end{array}$ & $\begin{array}{l}\text { H. } 22,6 ; \\
\text { 1. } 3,4 ; \\
\text { e. } 6,95\end{array}$ & $\begin{array}{l}\text { Textile sur devant du } \\
\text { clou, dos, bras, mains, } \\
\text { côtés de l'habit et } \\
\text { arrière de la coiffe. Voir } \\
\text { relevé (Thomas, 2012, } \\
\text { fig. 5) ; « filaments » sur } \\
\text { l'ensemble ; mention des } \\
\text { textiles : exp. Barcelone, } \\
\text { 2000, n } 55 \text {; Thomas } \\
2012 \text {; Garcia-Ventura } \\
2012 \text {, p. } 237\end{array}$ & $\begin{array}{l}\operatorname{Lin}^{47} ; \text { très } \\
\text { minéralisé, } \\
\text { sans } \\
\text { souplesse }\end{array}$ & $\begin{array}{l}\text { Toile } \\
\text { équi- } \\
\text { librée }\end{array}$ & $\begin{array}{l}\text { OX-OY : fil } \\
\text { retors de } \\
\text { torsion } \mathrm{S} \text { de } \\
\text { deux bouts } \mathrm{Z}\end{array}$ & $\begin{array}{l}\text { OX : } 0.32- \\
0.38 \\
\text { OY : } 0.32- \\
0.38\end{array}$ & $\begin{array}{l}\text { OX : } 16-18 \text { fils } / \mathrm{cm} \\
\text { OY : } 12-14 \text { fils } / \mathrm{cm}\end{array}$ \\
\hline 3 & $\begin{array}{l}\mathrm{AO} 77 \\
=85 ; \mathrm{CAC} 155\end{array}$ & $\begin{array}{l}\text { dieu } \\
\text { agenouillé }\end{array}$ & $\begin{array}{l}\text { H. } 20,2 \\
\text { 1. } 3,25 \\
\text { e. } 7,25\end{array}$ & $\begin{array}{l}\text { Textile sur devant } \\
\text { (milieu et sommet) } \\
\text { du clou, barbe, visage, } \\
\text { arrière de la coiffe et } \\
\text { du chignon côté droit, } \\
\text { épaules, bras, mains, } \\
\text { jambes, pieds, postérieur } \\
\text { et restes infimes épars ; } \\
\text { « filaments » sur } \\
\text { l'ensemble ; mention des } \\
\text { textiles : exp. Barcelone, } \\
\text { 2000, n } 55 \text {; Thomas ; } \\
\text { 2012, p. 151, note } 2 \text {; } \\
\text { Garcia-Ventura } 2012, \\
\text { p. } 237\end{array}$ & $\begin{array}{l}\text { Lin }{ }^{47} ; \\
\text { minéralisé, } \\
\text { ayant gardé } \\
\text { une certaine } \\
\text { souplesse (et } \\
\text { sa couleur } \\
\text { par endroits) }\end{array}$ & $\begin{array}{l}\text { Toile } \\
\text { équi- } \\
\text { librée }\end{array}$ & $\begin{array}{l}\text { OY : fils } \\
\text { simples de } \\
\text { torsion } \mathrm{Z} \\
\text { OX : fils retors } \\
\text { de torsion } \mathrm{S} \text { de } \\
\text { deux bouts } \mathrm{Z}\end{array}$ & $\begin{array}{l}\text { OX : } 0.15- \\
0.20 \\
\text { OY }: 0.15- \\
0.20\end{array}$ & $\begin{array}{l}\text { OX-OY : } 20 \text { fils } / \mathrm{cm} \\
\text { en moyenne }\end{array}$ \\
\hline 4 & $\begin{array}{l}\text { AO } 260 \\
=\text { Tello } 435\end{array}$ & $\begin{array}{l}\text { dieu } \\
\text { agenouillé }\end{array}$ & $\begin{array}{l}\text { H. } 21,2 ; \\
\text { l. } 4,55 \\
\text { e. } 7,1\end{array}$ & $\begin{array}{l}\text { Textile aujourd'hui disparu } \\
\text { sauf vestiges infimes au } \\
\text { creux du mollet gauche; } \\
\text { tissu mentionné dans fiches } \\
\text { anciennes }\end{array}$ & Inexploitable & & & & \\
\hline 5 & $\begin{array}{l}\text { AO } 311 \\
=A O 2355 \\
=C A C 146 \\
\text { Tello } 437\end{array}$ & $\begin{array}{l}\text { dieu } \\
\text { agenouillé }\end{array}$ & $\begin{array}{l}\text { H. } 29 \\
\text { l. } 8,5 \\
\text { e. } 13\end{array}$ & $\begin{array}{l}\text { Textile aujourd'hui disparu } \\
\text { mentionné dans fiches } \\
\text { anciennes }\end{array}$ & Disparu & & & & \\
\hline 6 & AO 312 & $\begin{array}{l}\text { dieu } \\
\text { agenouillé }\end{array}$ & $\begin{array}{l}\text { H. } 18,7 \\
\text { 1. } 3,95 ; \\
\text { e. } 6,45\end{array}$ & $\begin{array}{l}\text { Vestiges textiles infimes } \\
\text { à l'arrière de la nuque, } \\
\text { sur l'épaule gauche et au } \\
\text { sommet du clou ; traces } \\
\text { de tissu mentionnées } \\
\text { dans fiches anciennes ; } \\
\text { "filaments » à l'origine? }\end{array}$ & $\begin{array}{l}\text { Lin (?); très } \\
\text { minéralisé, } \\
\text { sans } \\
\text { souplesse }\end{array}$ & Toile & $\begin{array}{l}\text { Fil retors de } \\
\text { torsion } S \text { de } \\
\text { deux bouts } Z^{48}\end{array}$ & $0,25^{48}$ & 48 \\
\hline 7 & $\begin{array}{l}\text { MNB } 1362 \\
=\text { Tello } 443\end{array}$ & $\begin{array}{l}\text { dieu } \\
\text { agenouillé }\end{array}$ & $\begin{array}{l}\text { H. } 19,55 ; \\
\text { 1. } 3,65 ; \\
\text { e. } 6,8\end{array}$ & $\begin{array}{l}\text { Vestiges infimes : textile } \\
\text { sur le sommet du clou } \\
\text { et peut-être à la base du } \\
\text { chignon arrière, infimes } \\
\text { traces ; « filaments » à } \\
\text { l'origine? }\end{array}$ & $\begin{array}{l}\text { Lin (?); très } \\
\text { minéralisé, } \\
\text { sans } \\
\text { souplesse }\end{array}$ & Toile & $\begin{array}{l}\text { Fil retors de } \\
\text { torsion } \mathrm{S} \text { de } \\
\text { deux bouts } \mathrm{Z}^{48}\end{array}$ & $0.13-0.15$ & 48 \\
\hline
\end{tabular}




\begin{tabular}{|c|c|c|c|c|c|c|c|c|c|}
\hline & $\mathbf{N}^{\circ}$ d'inventaire $^{\prime}$ & $\begin{array}{l}\text { Type de } \\
\text { clou de } \\
\text { fondation }\end{array}$ & $\begin{array}{l}\text { Dimensions } \\
\text { en cm }\end{array}$ & $\begin{array}{l}\text { Remarques/observations } \\
\text { dans le cadre de cette } \\
\text { étude }\end{array}$ & Textile/ état & Armure & Torsion de fils & Diamètre & Réduction \\
\hline 8 & MNB 1365 & $\begin{array}{l}\text { dieu } \\
\text { agenouillé }\end{array}$ & $\begin{array}{l}\text { H. } 19,8 ; \\
\text { l. } 4,6 ; \\
\text { e. } 6,75\end{array}$ & $\begin{array}{l}\text { Textile mentionné } \\
\text { dans fiches anciennes, } \\
\text { aujourd'hui disparu } \\
\text { sauf vestiges infimes au } \\
\text { niveau du bras gauche; } \\
\text { "filaments" }\end{array}$ & $\begin{array}{l}\text { Lin (?); très } \\
\text { minéralisé, } \\
\text { sans souplesse }\end{array}$ & Toile & $\begin{array}{l}O X-O Y \text { : fils } \\
\text { retors de torsion } \\
S \text { de deux bouts } \\
Z\end{array}$ & $\begin{array}{l}O X: 0,21^{49} \\
O Y: 0,33\end{array}$ & 48 \\
\hline 9 & $\begin{array}{l}\text { MNB } 1374 \\
=\text { CAC } 160\end{array}$ & $\begin{array}{l}\text { taureau } \\
\text { couché }\end{array}$ & H. 22 & $\begin{array}{l}\text { Textile en bas à gauche } \\
\text { du clou sur le devant } \\
\text { et sur l'échine droite à } \\
\text { l'arrière ; « filaments » }\end{array}$ & $\begin{array}{l}\text { Lin (?); très } \\
\text { minéralisé, } \\
\text { sans } \\
\text { souplesse }\end{array}$ & $\begin{array}{l}\text { Toile } \\
\text { équi- } \\
\text { librée }\end{array}$ & $\begin{array}{l}\text { OX-OY : fils } \\
\text { retors de } \\
\text { torsion } \mathrm{S} \text { de } \\
\text { deux bouts } \mathrm{Z}\end{array}$ & $\begin{array}{l}\text { OX : } 0.39- \\
0.40 \\
\text { OY }: 0.32\end{array}$ & $\begin{array}{l}\text { OX-OY : } 14-16 \text { fils / } \\
\text { cm en moyenne }\end{array}$ \\
\hline 10 & $\begin{array}{l}\text { MNB } 1377 \\
=\text { CAC } 159\end{array}$ & $\begin{array}{l}\text { taureau } \\
\text { couché }\end{array}$ & $\begin{array}{l}\text { H. } 22,2 ; \\
\text { l. } 10,6 ; \\
\text { e. } 4,75\end{array}$ & $\begin{array}{l}\text { Textile au niveau } \\
\text { de l'échine gauche } \\
\text { de l'animal et sur le } \\
\text { clou ; « filaments » sur } \\
\text { l'ensemble }\end{array}$ & $\begin{array}{l}\text { Lin (?); très } \\
\text { minéralisé, } \\
\text { sans } \\
\text { souplesse }\end{array}$ & $\begin{array}{l}\text { Toile } \\
\text { équi- } \\
\text { librée }\end{array}$ & $\begin{array}{l}\text { OX-OY : fils } \\
\text { retors de } \\
\text { torsion } \mathrm{S} \text { de } \\
\text { deux bouts } \mathrm{Z}\end{array}$ & $\begin{array}{l}\text { OX : } 0.35 \\
\text { OY : } 0,35\end{array}$ & $\begin{array}{l}\text { OX-OY : } 20 \text { fils } / \mathrm{cm} \\
\text { en moyenne }\end{array}$ \\
\hline 11 & $M N B 1380$ & $\begin{array}{l}\text { dieu } \\
\text { agenouillé }\end{array}$ & H. 19,3 & $\begin{array}{l}\text { Tissu (aujourd'hui disparu) } \\
\text { d'après photographies avant } \\
\text { restauration à Nancy en } \\
1987 \text {; «filaments» }\end{array}$ & Disparu & & & & \\
\hline 12 & $\begin{array}{l}\text { MNB } 1384 \\
=1996-15 \\
\text { (Lyon) }\end{array}$ & $\begin{array}{l}\text { dieu } \\
\text { agenouillé }\end{array}$ & $\begin{array}{l}\text { H. } 19,7 ; \\
\text { m. } 4,6 ; \\
\text { e. } 6,5\end{array}$ & $\begin{array}{l}\text { Textiles sur le devant } \\
\text { du clou en bas, sur les } \\
\text { épaules, les bras, le bas } \\
\text { de la barbe, les côtés } \\
\text { de l'habit, la jambe } \\
\text { et le pied gauche, dans } \\
\text { le dos ; « filaments »; } \\
\text { mention des textiles: } \\
\text { Rashid, 1983, n } 97 \text {; } \\
\text { Garcia-Ventura, } 2012 \text {, } \\
\text { p. } 237\end{array}$ & $\begin{array}{l}\operatorname{Lin}^{47} \\
\text { très } \\
\text { minéralisé, } \\
\text { sans } \\
\text { souplesse }\end{array}$ & $\begin{array}{l}\text { Toile } \\
\text { équi- } \\
\text { librée }\end{array}$ & $\begin{array}{l}\text { OX-OY : fils } \\
\text { retors de } \\
\text { torsion } \mathrm{S} \text { de } \\
\text { deux bouts } \mathrm{Z}\end{array}$ & $\begin{array}{l}\text { OX : } 0.28- \\
0.33 \\
\text { OY }: 0.33- \\
0.35\end{array}$ & $\begin{array}{l}\text { OX-OY : } 12 \text { fils } / \mathrm{cm} \\
\text { en moyenne }\end{array}$ \\
\hline \multicolumn{10}{|c|}{ Musée archéologique, Istanbul } \\
\hline 13 & EŞEM 1572 & $\begin{array}{l}\text { dieu } \\
\text { agenouillé }\end{array}$ & & $\begin{array}{l}=\text { AO } 26678(\text { ?); } \\
29144 \text { (?); Istanbul (?); } \\
\text { mention des textiles : } \\
\text { Rashid, } 1983, \mathrm{n}^{\circ} 82\end{array}$ & Non vérifié & & & & \\
\hline 14 & EŞEM 1721 & $\begin{array}{l}\text { dieu } \\
\text { agenouillé }\end{array}$ & & $\begin{array}{l}\text { Mention des textiles : } \\
\text { Rashid, } 1983, \mathrm{n}^{\circ} 84\end{array}$ & Non vérifié & & & & \\
\hline \multicolumn{10}{|c|}{ Lieu de conservation non identifié } \\
\hline 15 & $T G 15 a$ & $\begin{array}{l}\text { dieu } \\
\text { agenouillé }\end{array}$ & & $\begin{array}{l}\text { Textiles visibles: } \\
\text { Genouillac, 1934, pl. 87, } 1\end{array}$ & & & & & \\
\hline 16 & $T G 16 a$ & $\begin{array}{l}\text { dieu } \\
\text { agenouillé }\end{array}$ & & $\begin{array}{l}=\text { AO } 26678 \text { (?); } \\
29144 \text { (?); Istanbul (?); } \\
\text { textiles visibles : Genouillac, } \\
\text { 1936, pl. } 87,2 ; \text { Garcia- } \\
\text { Ventura, } 2012, \text { p. } 237\end{array}$ & & & & & \\
\hline 17 & $T G 17 a$ & $\begin{array}{l}\text { dieu } \\
\text { agenouillé }\end{array}$ & & $\begin{array}{l}=\text { AO } 26678 \text { (?); } \\
29144 \text { (?); Istanbul (?); } \\
\text { textiles visibles : Genouillac, } \\
1934, \text { pl. } 87,3\end{array}$ & & & & & \\
\hline
\end{tabular}




\section{Tableau II. Autres clous de fondation de la II $^{\mathrm{e}}$ dynastie de Lagash ayant pu porter des textiles}

\begin{tabular}{|c|c|c|c|c|c|}
\hline & $\mathbf{N}^{\circ}$ d'inventaire & $\begin{array}{l}\text { Type de clou } \\
\text { de fondation }\end{array}$ & $\begin{array}{l}\text { Dimensions } \\
\text { en } \mathbf{c m}\end{array}$ & Provenance & Remarques/références \\
\hline \multicolumn{6}{|c|}{ Musée du Louvre, Paris } \\
\hline 1 & $\begin{array}{l}\text { AO } 258 \\
=\text { CAC } 157\end{array}$ & porteur de corbeille & H. 24,4 & Tello ; fouilles Sarzec & $\begin{array}{l}\text { Pas de textile visible ni attesté } \\
\text { dans la documentation }\end{array}$ \\
\hline 2 & AO 25581 & dieu agenouillé & H. 19,5 & Tello ; fouilles Sarzec & $\begin{array}{l}\text { Pas de textile visible ni attesté } \\
\text { dans la documentation ; « filaments» }\end{array}$ \\
\hline 3 & $\begin{array}{l}\text { AO } 26678 \\
=\text { CAC } 158\end{array}$ & porteur de corbeille & H. 24 & Tello ; ancien fonds & $\begin{array}{l}\text { Pas de textile visible ni attesté } \\
\text { dans la documentation } \\
=\text { TG } 15,16 \text { ou } 17(?)^{50} \text { ou fouilles Cros } \\
\text { (Cros } \text { et al., 1914, p; } 66 \text { et } 282 \text { )? }\end{array}$ \\
\hline 4 & $\begin{array}{l}\text { AO } 29144 \\
=\text { CAC } 147 \\
\text { Tello } 445\end{array}$ & dieu agenouillé & H. 19,4 & $\begin{array}{l}\text { Tello ; fouilles } \\
\text { Genouillac? }\end{array}$ & $\begin{array}{l}\text { Pas de textile visible ni attesté } \\
\text { dans la documentation } \\
=\text { «AO ou TG } 445 » \text { dans Suter, } 2000 \text {, p. } 317 \\
\text { (FG } 20 \text { ); Garcia-Ventura, } 2012 \text {, p. } 237 \\
=\text { TG } 15 \text {, TG } 16 \text { ou TG } 17(?)^{50}\end{array}$ \\
\hline 5 & $\begin{array}{l}\text { MNB } 1371 \\
=\text { CAC } 162\end{array}$ & taureau couché & H. 25,6 & Tello, tell M & $\begin{array}{l}\text { Pas de textile visible ni attesté } \\
\text { dans la documentation }\end{array}$ \\
\hline \multicolumn{6}{|c|}{ Museo Barracco, Rome } \\
\hline 6 & MB 45 & dieu agenouillé & H. 18 & Coll. Hakky Bey & $\begin{array}{l}\text { Pas de textile visible sur photographie ; } \\
\text { Van Buren, 1931, p. 16, pl. VII, fig. } 13\end{array}$ \\
\hline \multicolumn{6}{|c|}{ Vorderasiatiches Museum, Berlin } \\
\hline 7 & VA 3023 & dieu agenouillé & H. 17,4 & $?$ & $\begin{array}{l}\text { Textile (?) ; Van Buren, 1931, p. } 15 \text {; } \\
\text { Suter, } 2000, \mathrm{n}^{\circ} 27\end{array}$ \\
\hline 8 & VA 3056 & dieu agenouillé & & $?$ & $\begin{array}{l}\text { Perdu pendant la guerre }(1939-1945) \text {; } \\
\text { Van Buren, 1931, p. } 15 \text {; Suter, 2000, nº } 28\end{array}$ \\
\hline \multicolumn{6}{|c|}{ Nelson Atkins Museum of Art, Kansas City } \\
\hline 9 & $30-1 / 50$ & dieu agenouillé & H. 20,3 & Nelson Trust & $\begin{array}{l}\text { Pas de textile visible; Aruz, 2003, fig. 312a, } \\
\text { p. } 439\end{array}$ \\
\hline \multicolumn{6}{|c|}{ Walters Art Museum, Baltimore } \\
\hline 10 & 54.790 & dieu agenouillé & & $?$ & Textile (?) ; Suter, $2000, n^{\circ} 31$ \\
\hline \multicolumn{6}{|c|}{ Pierpont Morgan Library, New York City } \\
\hline 11 & $\begin{array}{l}\text { AZ.145 } \\
\text { MLC2388 }\end{array}$ & dieu agenouillé & H. 19,5 & $?$ & $\begin{array}{l}\text { Pas de textile visible ; Suter, } 2000, \mathrm{n}^{\circ} 30 \text {; } \\
\text { Aruz, 2003, fig. } 312 \text { b, p. } 439\end{array}$ \\
\hline \multicolumn{6}{|c|}{ Yale Babylonian Collection, University of Yales } \\
\hline 12 & $?$ & dieu agenouillé & H. 20,5 & $?$ & Textile (?) ; Van Buren, 1931, p. 16, note 2 \\
\hline \multicolumn{6}{|c|}{ British Museum, Londres } \\
\hline 13 & BM 91056 & dieu agenouillé & H. 18,9 & $?$ & $\begin{array}{l}\text { Pas de textile visible sur photographie ; } \\
\text { Van Buren, 1931, p. } 15\end{array}$ \\
\hline 14 & BM 91057 & dieu agenouillé & H. 19 & $?$ & $\begin{array}{l}\text { Pas de textile visible sur photographie ; } \\
\text { Van Buren, 1931, p. } 15\end{array}$ \\
\hline 15 & BM 91058 & dieu agenouillé & H. 17,3 & $?$ & $\begin{array}{l}\text { Pas de textile visible sur photographie ; } \\
\text { Van Buren, 1931, p. } 15\end{array}$ \\
\hline 16 & BM 96566 & dieu agenouillé & H.16,05 & $?$ & $\begin{array}{l}\text { Pas de textile visible sur photographie } \\
\text { Van Buren, 1931, p. } 15\end{array}$ \\
\hline 17 & BM 102613 & dieu agenouillé & H. 14,2 & $?$ & $\begin{array}{l}\text { Pas de textile visible sur photographie } \\
\text { Van Buren, 1931, p. } 15 \text {, pl. VII, fig. } 12\end{array}$ \\
\hline
\end{tabular}




\begin{tabular}{|c|c|c|c|c|c|}
\hline & $\mathrm{N}^{\circ}$ d'inventaire & $\begin{array}{l}\text { Type de clou } \\
\text { de fondation }\end{array}$ & $\begin{array}{l}\text { Dimensions } \\
\text { en cm }\end{array}$ & Provenance & Remarques/références \\
\hline \multicolumn{6}{|c|}{ Musée de Mariemont, Mariemont } \\
\hline 18 & 139 & dieu agenouillé & & Tello & Textile (?); Suter, $2000, \mathrm{n}^{\circ} 33$ \\
\hline \multicolumn{6}{|c|}{ Israël Museum, Jérusalem } \\
\hline 19 & 71.23 .299 & dieu agenouillé & & Anc. coll. Brummer & Textile (?); Suter, 2000, n 32 \\
\hline \multicolumn{6}{|c|}{ Iraq Museum, Bagdad } \\
\hline 20 & IM 6954 & dieu agenouillé & & $\begin{array}{l}\text { Tello, tell A, sous dallage } \\
\text { cour A }\end{array}$ & Textile (?); Suter, $2000, n^{\circ} 18$ \\
\hline \multicolumn{6}{|c|}{ Musée archéologique, Istanbul } \\
\hline 21 & EŞEM 491 & dieu agenouillé & & Tello & $\begin{array}{l}\text { Textile disparu d'après photographie } \\
\text { récente ; Suter, } 2000, n^{\circ} 15\end{array}$ \\
\hline 22 & EŞEM 492 & dieu agenouillé & & Tello & Textile (?) ; Suter, 2000, n 14 \\
\hline 23 & EŞEM 1524 & dieu agenouillé & & Tello & Textile (?) ; Suter, 2000, n $^{\circ} 10$ \\
\hline 24 & EŞEM 1574 & dieu agenouillé & & Tello & Textile (?) ; Suter, $2000, \mathrm{n}^{\circ} 12$ \\
\hline 25 & EŞEM 6024 & dieu agenouillé & & Tello & Textile (?) ; Suter, $2000, \mathrm{n}^{\circ} 16$ \\
\hline 26 & EŞEM 6504 & dieu agenouillé & & Tello & Textile (?) ; Suter, $2000, \mathrm{n}^{\circ} 17$ \\
\hline
\end{tabular}

\section{Notes}

1. Rappelons brièvement que ces clous fichés dans le sol, à l'intérieur de logettes en briques ménagées dans les fondations des édifices, avaient pour fonction de délimiter l'espace sacré, mais aussi d'ancrer au sol les forces malfaisantes (voir Van Buren, 1931, p. 11-12 ; Genouillac, 1934, p. 90 ; Parrot, 1948 , p. 202).

2. Ces clous ont été réalisés en fonte pleine, en cuivre quasiment pur présentant une forte teneur en arsenic (plus de $1 \%$ en masse) d'après ceux qui furent analysés. Analyses du métal : Berthelot, 1889 ; Hauptmann, 2004, p. 147 ( $\mathrm{n}^{\circ}$ 2491-2503, 2505-2508) et, pour le clou inventorié AO 75 , D. Bourgarit (C2RMF). Compte tenu des ressources locales, il s'agissait d'une dépense importante, significative de la valeur accordée à ces objets.

3. Malgré de fortes similitudes au-delà même du type auquel ils appartiennent, ces clous semblent issus de plusieurs moules d'après certaines différences déjà remarquées par Heuzey, 1902, p. 304.

4. Une femme selon certains (par exemple Van Buren, 1931, p. 16), mais plus vraisemblablement le roi bâtisseur comme sur d'autres exemples jusqu'au I ${ }^{\mathrm{er}}$ millénaire (par exemple Assurbanipal : British Museum, inv. BM 90864).

5. En excluant 3 clous non identifiés qui pourraient correspondre à d'autres comptés par ailleurs (tableau I, 15-17).

6. Voire 16 si 3 clous publiés par Genouillac, 1934, pl. 87, 1-3 (tableau I,
15-17) ne sont pas à déduire de 2 clous aujourd'hui conservés à Istanbul (tableau I, 13-14).

7. Sarzec, 1884 , p. 242 ; Cros, 1910, p. 66 et 282 ; Genouillac, 1912, p. 90 ;

Parrot, 1948, p. 202.

8. AO 76, AO 77, MNB 1374, MNB 1377 , MNB 1384 et l'on détecte des vestiges infimes mais visibles au microscope sur MNB 1362, MNB 1365 et AO 312 et, de manière excessivement lacunaire, sur AO 260 et AO 75. Par ailleurs, bien que n'ayant pu être vus dans le cadre de cette étude, 2 clous du Musée archéologique d'Istanbul

conserveraient encore des textiles en surface.

9. Certains portent les marques de traitement excessif vraisemblablement à l'acide (voir AO 29144).

10. Communication de Luc Robbiola.

11. Goossens, 1958, p. 157. Sarzec, 1884, p. 243, supposait d'ailleurs que les figurines qu'il avait découvertes aient été des remplois.

12. Par ailleurs, seuls 3 clous ayant assurément porté des textiles ne relevaient pas du type du dieu agenouillé.

13. Voir des clous en forme de porteur de corbeille provenant de Nippur et conservés au Musée national de Bagdad et à l'Oriental Institute de Chicago (Basmachi, 1972, p. 140, 400, fig. 101 ; Garcia-Ventura, 2008 et 2012, p. 241).

14. Garcia-Ventura, 2012, p. 246.

15. Thomas, 2012.

16. Catalogue à paraître.

17. Quoique minéralisés (à différents degrés : voir tableau I) et bien qu'ils ne soient pas représentatifs de toutes les catégories textiles connues par les textes et suggérées par l'iconographie.

18. Avec la collaboration de Dominique Robcis et Noëlle Timbart que nous remercions vivement pour leur aide.

19. Dans le cadre de cet article, cela n'a pas été le cas des clous de même type mais aux vestiges trop infimes, ainsi que de deux clous en forme de taureau car relevant d'un autre type.

20. Moulherat, 2008.

21. La plupart des spécialistes travaillant sur les fibres minéralisées procèdent aux identifications à partir de l'observation de cassures rendant toute mesure de diamètre précise impossible.

22. Analyses effectuées au musée du quai Branly sur un MEB HITACHI TM 3000.

23. La plante appauvrit considérablement les sols qu'il faut régénérer constamment, alors que les sols mésopotamiens devaient être massivement exploités pour les plantations de céréales. Il est possible que le lin mésopotamien ait appartenu à la catégorie des plantes semidomestiques sur des emplacements ponctuellement disponibles ou sur de petites parcelles au sol enrichi notamment en eau selon des pratiques horticoles et non pas intensives (Breniquet, 2008, p. 87-88).

24. Waetzoldt, 1972 ; Joannès, 2001 p. 472-473. Le lin fait toutefois partie des premières plantes domestiquées par l'homme au Moyen-Orient (en Mésopotamie, vestiges mis au jour à Tell es Sawan et Choga Mami (Irak) vers $6000 / 5000$ avant J.-C.) 
25. Comme on le sait des textiles et vêtements de lin les plus précieux (Joannès, 2001, p. 472-473).

26. Waetzoldt, 1983 , p. 67-68 ; GarciaVentura, 2012, p. 246. Le lin serait en outre attesté dans les tombes royales d'Ur vers 2500 avant J.-C. (Woolley, 1934, p. 143).

27. En collaboration avec le laboratoire IPANEMA (USR3461 CNRS/ministère de la Culture et de la Communication Synchrotron SOLEIL), un master dans le cadre du labex Patrima prévoit d'approfondir la question des couleurs (encadrement L. Bertrand - A. Thomas).

28. Joannès, 2001, p. 473. Les textes cunéiformes mentionnent cependant plusieurs couleurs pour des étoffes de lin, ce qui démontre que le lin pouvait alors être teint, du moins en Mésopotamie.

29. Le clou inventorié AO 77 est suffisamment bien conservé pour que le diamètre des fils soit avéré.

30. À ce jour, ces filaments ne sont attestés que sur ces clous de fondation, à l'exception d'une statuette d'époque amorrite (musée du Louvre, inv. AO 15704) à la surface de laquelle des traces comparables apparaissent. Elle représente d'ailleurs un homme agenouillé dans une attitude très comparable à celle des figurines de dieu, quoiqu'il ne tienne aucun clou et que la pièce ne soit pas un dépôt de fondation mais un objet de dévotion.

31. Sur les 12 de la collection du musée du Louvre, seuls 4 clous qui ont fait l'objet d'un traitement important en surface ne portent pas ou plus traces de ces « filaments» (voir tableau I).

32. Voir tableaux I et II.

33. Communication de Dominique Robcis sur l'observation de traces de feutre sur d'autres objets.

34. Communication de Luc Robbiola (Laboratoire TRACES (CNRS UMR 5608), université Toulouse Jean-Jaurès).

35. Edzard, 1997, p. 39, E3/1.1.7.StC, iii 8-10) ; p. 44, E3/1.1.7.StD, iii 11)-12); p. 47, E3/1.1.7.StF, iii 3) -5)

36. On connaît ainsi des habits dont l'étoffe aurait été couverte de produits destinés à lui donner un aspect particulier (Van de Mieroop, 1987, p. 42 par exemple). 37. Oppenheim, 1949 ; Winter, 1992 ; Sigrist, 2004.

38. Aruz, 2003, p. 439

39. En effet, les rites de fondation mésopotamiens se référeraient au temple d'Enki à Nippur (Van Buren, 1952, p. 293) et la position de la figurine est proche de celle des acolytes de ce dieu sur des sceaux akkadiens (voir par exemple, Schlossman, 1976, p. 11, 13 ; musée du Louvre, inv. AO 22303).

40. Ellis, 1968, p. 22-26, 31 ; Suter, 2000, p. 395 ; Garcia-Ventura, 2012, p. 244, 250.

41. Voir par exemple : musée du Louvre, inv. AO 19825, bien que le taureau y figure paré de bijou mais non vêtu.

42. Van Buren, 1931, p. 13.

43. Comme cela a pu être mis en évidence à Nippur : Ellis, 1966, p. 68.
44. Genouillac, 1912, pl. II, fig. 6659. En revanche, aucune trace n'a été détectée sur les nombreux clous de fondation en argile de la $\mathrm{II}^{\mathrm{e}}$ dynastie de Lagash, les textiles étant peut-être réservés aux clous figurés, soit incarnés.

45. Ellis, 1968, p. 184 ; des perles ont ainsi été retrouvées à proximité de figurines de fondation à Nippur (Ellis, 1966, p. 68) et l'on connaît plusieurs dépôts de fondation comportant des bijoux (par exemple à Mari ou à Khorsabad : musée du Louvre, inv. AO 29515, AO 29517, AO 29518 ; AO 30266-AO 30273).

46. $\mathrm{CAC}=$ Heuzey, 1902

47. Prélèvements examinés au MEB.

48. Trop peu de matière subsistante pour distinguer trame et chaîne, ainsi que pour mesurer le nombre de fils au $\mathrm{cm}$.

49. Fil probablement dégradé.

50. AO 26678 et AO 29144 pourraient correspondre à TG 15, TG 16 ou TG 17 (?), car issus des fouilles Genouillac (assurément pour l'un et hypothétiquement pour $\mathrm{AO}$ 26678, mais leur surface est aujourd'hui très restaurée sans aucune trace textile).

\section{Références bibliographiques}

Aruz J. (dir.), 2003, Art of the First Cities. The Third Millenium B.C. from the Mediterranean to the Indus, New York, The Metropolitan Museum of Art, Yale University Press.

Basmachi F., 1972, Treasures of the Iraq Museum, Bagdad.

Berthelot M., 1889, Introduction à l'étude de la chimie des anciens et du Moyen Âge, Paris, Georges Steinheil.

Breniquet C., 2008, Essai sur le tissage en Mésopotamie des premières communautés sédentaires au milieu du III millénaire avant J.-C., Paris, De Boccard.

Cros G., Heuzey L. et Thureau-Dangin F., 1910, Nouvelles fouilles de Tello, Paris, Leroux.

Edzard D. O., 1997, Gudea and His Dynasty. The Royal Inscriptions of Mesopotamia, 3/1, Toronto, Buffalo, London, University of Toronto Press.

Ellis R. S., 1966, Foundation Deposits in Ancient Mesopotamia, New Haven.

Exp. Barcelone, 2000, La fundacion de la ciudad, Mesopotamia, Grecia, Roma, Barcelone, Centre de cultura contemporania de Barcelona.

Garcia-Ventura A., 2008, "Neo-Sumerian Textiles Wrappings. Revisiting some Foundation Figurines from Nippur", Zeitschriftfür Orient-Archäologie, 1, p. 247-252.

Garcia-Ventura A., 2012, "The Emperor's New Clothes: Textiles, Gender and Mesopotamian Foundation Figurines", Altorientalische Forschungen, 39-2, p. 235-253.

Genouillac H. de, 1912, Inventaire des tablettes de Tello conservées au musée impérial ottoman III, Paris, Ernest Leroux.

Genouillac H. de, 1934, Fouilles de Telloh II, Paris, Mission archéologique du musée du Louvre, P. Geuthner.
Goossens G., 1958, « Les recherches historiques à l'époque néobabylonienne ", Revue d'assyriologie et d'archéologie orientale, 52, p. 150-159.

Hauptmann H. et Pernicka E. (dir.), 2004, Die Metallindustrie Mesopotamiens von den Anfängen bis zum 2. Jahrtausend v. Chr., Rahden, Verlag Marie Leidorf (OrientArchäologie 3).

Heuzey L., 1902, Catalogue des Antiquités chaldéennes, Paris, musée du Louvre.

Joannès F., 2001, Dictionnaire de la civilisation mésopotamienne, Paris, Bouquins, Robert Laffont.

Moulherat C., 2008, « L'archéologie des textiles, une nouvelle discipline au service de la connaissance et de la compréhension des sociétés humaines ", L'actualité chimique, Chimie et patrimoine culturel, 318, p. 30-34.

Oppenheim A. L., 1949, "The Golden Garments of the Gods", Journal of Near Eastern Studies, VIII, 3, p. 172-193.

Parrot A., 1948, Tello, vingt campagnes de fouilles (1877-1933), Paris, Albin Michel.

Rashid S. A., 1983, Gründungsfiguren im Iraq, Munich, C. H. Beck.

Sarzec E. de, 1884-1912, Découvertes en Chaldée II, Paris, Ernest Leroux.

Schlossman B. L., 1976, "Two Foundation Figurines", Ancient Mesopotamian Art and Selected Texts, New York, The Pierpont Morgan Library, p. 9-21.

Sigrist M., 2004, « Fabrication d'images », in Waetzoldt H. (dir.) Von Sumer nach Eblaundzurück. Festschrift - Giovanni Pettinato, Heidelberg, Heidelberg Orient Verlag, p. 257-283.

Suter C., 2000, Gudea's Temple Building. The Representation of an Early Mesopotamian Ruler in Text and Image, Groningen, Styx.

Thomas A., 2012, « Restes textiles sur un clou de fondation de Gudea. Étude préliminaire ", Paléorient, 38, 1, p. 151-159.

Van Buren E. D., 1931, Foundation Figurines and Offerings, Berlin, Hans Schoetz \& Co.

Van Buren E. D., 1952, "Foundation Rites for a New Temple”, Orientalia, 21, p. 293-306.

Van de Mieroop M., 1987, "Crafts in the Early Isin period, a study of the Isin craft Archive from the reigns of IsbiErra and Su-Ilisu", Orientalia Lovaniensia Analecta, 24.

Waetzoldt H., 1972, Untersuchungen zur neusumerischen Textilindustrie, Rome, Studi economici e tecnologici.

Waetzoldt H., 1983, "Leinen", Reallexikon der Assyriologie, 6, p. 583-594.

Winter I., 1992, "Idols of the King, Royal Images as Recipients of Ritual Action in Ancient Mesopotamia”, Journal of Ritual Studies, 6/1, p. 13-42.

Woolley L., 1934, The Royal Cemetery, Ur Excavations, II, Londres et Philadelphie, British Museum et University Museum. 